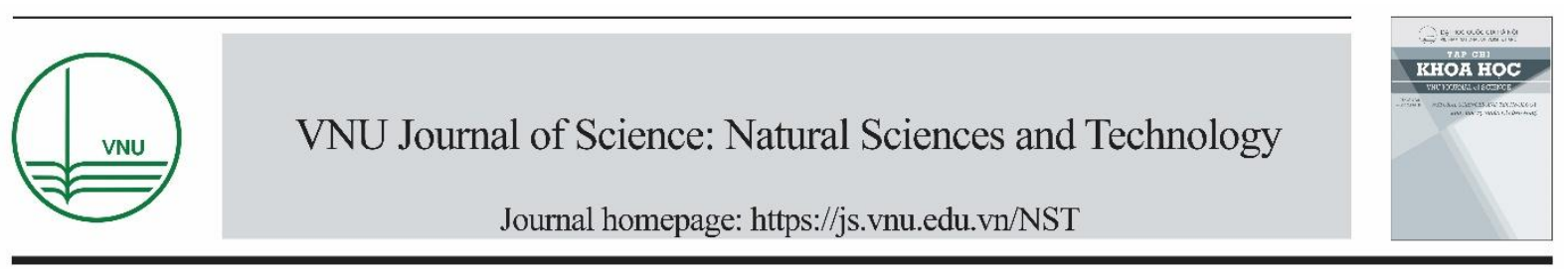

Original Article

\title{
Synthesis of SSZ-13 Zeolite in the Presence of N,N,N- Dimethylethylcyclohexyl Ammonium for Selective Catalytic Reduction of $\mathrm{NO}_{\mathrm{x}}$
}

\author{
Dang Van Long*, Le Thanh Son, Pham Dinh Trong \\ Faculty of Chemistry, VNU University of Science, Vietnam National University, Hanoi \\ 19 Le Thanh Tong, Hoan Kiem, Hanoi, Vietnam
}

Received 06 June 2019

Revised 14 December 2019; Accepted 17 December 2019

\begin{abstract}
Since $\mathrm{NO}_{x}$ emission requirements from stationary and mobile sources are more strictly regulated in the United States, Europe, and other countries; researchers have conducted many studies to improve the performance of selective catalytic reduction (SCR) catalysts to meet more and more stringent emission standards. Herein, we reported the synthesis of small pore zeolite (Cu)-SSZ-13 using $N, N, N$-dimethylethylcyclohexylammonium as the structure directing agent. The catalytic activity of the fresh and hydrothermal aged copper exchanged supported on SSZ-13 catalyst was investigated in the SCR of $\mathrm{NO}_{x}$ using $\mathrm{NH}_{3}$ as a reductant. Cu-SSZ-13 possessing a high SCR performance $\left(\mathrm{NO}_{\mathrm{x}}\right.$ conversion reached approximately $100 \%$ at $\left.250^{\circ} \mathrm{C}\right)$, and high hydrothermal stability in combination with an easy synthesis route is considered to be a potential catalyst for SCR application.
\end{abstract}

Keywords: Zeolite, SSZ-13, synthesis, SCR, $\mathrm{NO}_{\mathrm{x}}$.

\footnotetext{
* Corresponding author.

Email address: longdv@vnu.edu.vn

https://doi.org/10.25073/2588-1140/vnunst.4915
} 


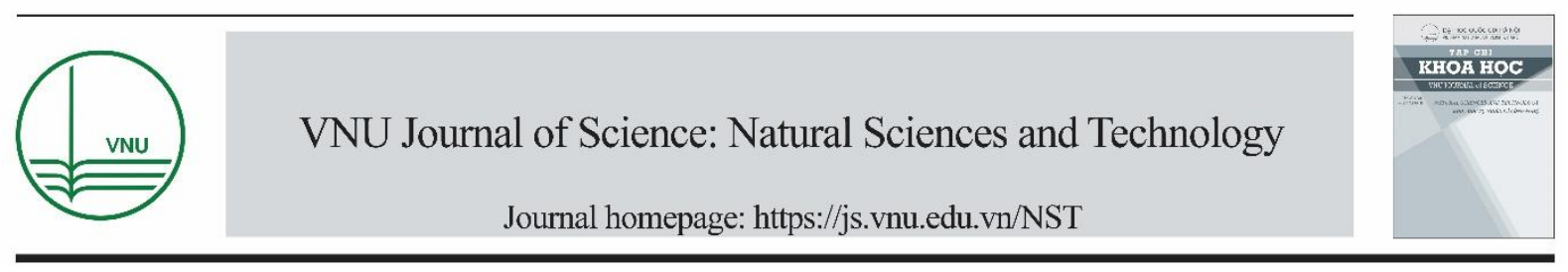

\title{
Tổng hợp zeolit SSZ-13 sử dụng chất định hướng cấu trúc $\mathrm{N}, \mathrm{N}, \mathrm{N}-$ Dimethylethylcyclohexyl ammonium ứng dụng trong phản ứng khử chọn lọc $\mathrm{NO}_{\mathrm{x}}$
}

\author{
Đặng Văn Long*, Lê Thanh Sơn, Phạm Đình Trọng \\ Khoa Hoá họ, Truờng Đại học Khoa học Tư nhiên, ĐHQGHN \\ 19 Lê Thánh Tông, Hà Nội, Việt Nam \\ Nhận ngày 06 tháng 6 năm 2019 \\ Chỉnh sửa ngày 14 tháng 12 năm 2019; Chấp nhận đăng ngày 17 tháng 12 năm 2019
}

\begin{abstract}
Tóm tắt: Công nghệ xúc tác khử chọn lọc (SCR) khí thải $\mathrm{NO}_{\mathrm{x}}$ từ các nhà máy và động cơ ô tô gần đây thu hút sự chú ý lớn của các nhà khoa học trên thế giới nhằm đáp ứng các tiêu chuẩn khí thải ngày càng nghiêm ngặt tại Hoa Kỳ, Châu Âu, và một số quốc gia khác. Trong nghiên cứu này, chúng tôi đưa ra phương pháp tổng hợp zeolit Chabazite mao quản nhỏ (SSZ-13) sử dụng chất tạo cấu trúc $N, N, N$-dimethylethylcyclohexylammonium. Hoạt tính xúc tác của xúc tác SSZ-13 trao đổi ion đồng (Cu-SSZ-13) trước và sau khi xử lý thủy nhiệt ở $750^{\circ} \mathrm{C}$ được nghiên cứu trong phản ứng SCR của $\mathrm{NO}_{x}$ sử dụng $\mathrm{NH}_{3}$ làm chất khử. Kết quả nghiên cứu cho thấy Cu-SSZ-13 cho độ chuyển hóa $\mathrm{NO}_{\mathrm{x}}$ cao trong phản ứng SCR $\left(\sim 100 \%\right.$ tại $\left.250^{\circ} \mathrm{C}\right)$, độ bền thủy nhiệt cao, kết hợp với phương pháp tổng hợp dễ dàng từ chất tạo cấu trúc có giá thành tương đối thấp được coi là chất xúc tác tiềm năng cho ứng dụng công nghệ SCR.
\end{abstract}

Tù khóa: Zeolit, SSZ-13, SCR, $\mathrm{NO}_{x}$.

\section{Mở đầu}

Các zeolit mao quản (MQ) nhỏ vòng tám cạnh (Eight-Membered Ring, 8MR), bao gồm các dạng silicoaluminophosphate và aluminosilicate như zeolit AEI, AFX, CHA,... gần đây đã nhận được sự quan tâm lớn ứng dụng làm chất xúc tác cho phản ứng chuyển hóa methanol thành olefin (MTO) [1-3] và chọn lọc khử xúc tác (Selective

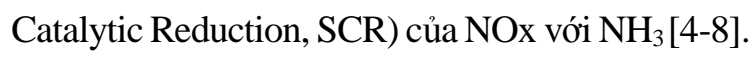
Các zeolit mao quản nhỏ (8MR) này đang được nghiên cứu rộng rãi do lợi thế đáng kể so với các zeolit mao quản trung bình và lớn bao gồm hoạt tính SCR cao, cải thiện độ bền thủy nhiệt, độ chọn lọc $\mathrm{N}_{2}$ cao hơn, hiệu suất hình thành sản phẩm phụ $\mathrm{N}_{2} \mathrm{O}$ giảm, và tăng khả năng chống ngộ độc hydrocacbon [9-16]. Chất xúc tác dựa trên zeolit CHA được thương mại hóa vào năm

\footnotetext{
* Tác giả liên hệ.

Địa chi email: longdv@vnu.edu.vn

https://doi.org/10.25073/2588-1140/vnunst.4915
} 
2010 và hiện đang là sự lựa chọn phổ biến nhất cho công nghệ khử xúc tác chọn lọc $\mathrm{NH}_{3}-\mathrm{SCR}$ của $\mathrm{NOx}$ trong việc kiểm soát lượng khí thải từ xe hơi [7,12]. Zeolit SSZ-13 (cấu trúc khung dạng CHA) chứa ion kim loại đồng với mật độ $\mathrm{T}=\mathrm{Si} / \mathrm{Al}$ của cấu trúc thấp (nghĩa là vật liệu có độ xốp cao, $15,1 \mathrm{~T}$ nguyên tử trên $1000 \AA^{3}$ ) và hệ thống kênh mao quản 3 chiều dựa trên các đơn vị cấu trúc thứ cấp D6R (double 6-rings, 2 vòng 6 cạnh) đều có hoạt tính SCR tốt và độ bền thủy nhiệt cao [17]. SSZ-13 thuộc nhóm không gian R3m (trigonal) [18], bao gồm ba vòng kép 6 cạnh (D6Rs), ba hốc ellipsoidal lớn (7 Âx11 A) và chín cửa sổ mao quản vòng 8 cạnh trong một ô mạng cơ sở gồm 36 nguyên tử $\mathrm{T}=\mathrm{Si} / \mathrm{Al}$ (Hình 1).

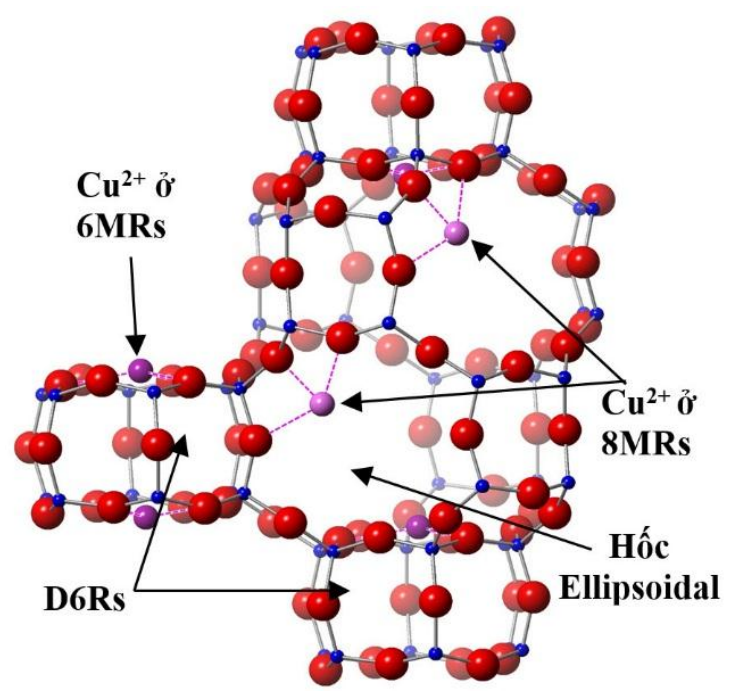

Hình 1. Cấu trúc zeolit SSZ-13 chứa ion đồng $(\mathrm{Cu}-$ SSZ-13). Các nguyên tử ôxy là các quả cầu màu đỏ, đồng là những quả cầu tím, và các nguyên tử $\mathrm{Si} / \mathrm{Al}$ là những quả cầu màu xanh lam. Ghi chú: 6/8MR=6/8-membered ring: vòng 6/8 cạnh; $D 6 R$ (double 6-ring): 2 vòng 6 cạnh; cage: hốc mao quản.

Một hạn chế của tổng hợp các vật liệu SSZ13 hàm lượng silic cao là chi phí tương đối cao của chất tạo cấu trúc $N, N, N$-trimethyl-1adamantammonium (TMAda ${ }^{+}$) được sử dụng để tổng hợp chúng [19,20]. Gần đây, G. Cao và cộng sự [21-23] công bố tổng hợp thủy nhiệt của chabazite hàm lượng $\mathrm{Si}$ cao với các tỷ lệ $\mathrm{Si} / \mathrm{Al}$ khác nhau bằng cách sử dụng $\mathrm{N}, \mathrm{N}, \mathrm{N}-$ dimethylethylcyclohexylammonium (DMECHA, Hình 2) làm chất định hướng cấu trúc (structure directing agent, SDA). Trong nghiên cứu này, chúng tôi sử dụng DMECHA làm chất tạo cấu trúc rẻ hơn TMAda để tổng hợp zeolit SSZ-13 với tỉ lệ $\mathrm{Si} / \mathrm{Al}$ cao $(\mathrm{Si} / \mathrm{Al}=11)$ trong môi trường kiềm. Vật liệu $\mathrm{Cu}-\mathrm{SSZ}-13$ được tổng hợp tiếp theo bằng phương pháp trao đổi ion và được nghiên cứu hoạt tính xúc tác trong phản ứng $\mathrm{SCR}$ của $\mathrm{NO}_{\mathrm{x}}$ bằng $\mathrm{NH}_{3}$.

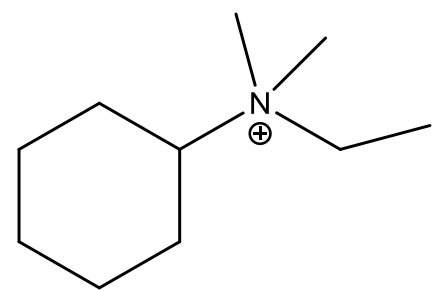

Hình 2. Công thức cấu tạo của chất định hướng cấu trúc DMECHA.

\section{Thực nghiệm và phương pháp nghiên cứu}

\subsection{Tổng hợp xúc tác}

Tổng hơp zeolit SSZ-13: Dung dịch gồm Banco $\mathrm{N}$ silicate $\left(8,9 \% \quad \mathrm{Na}_{2} \mathrm{O}, 28 \% \mathrm{SiO}_{2}, \mathrm{PQ}\right.$ corporation), $\mathrm{NaOH}$ (Fisher Scientific, 98\% wt) và nước đề ion hóa được khuấy ở nhiệt độ phòng trong khoảng 15 phút. Sau đó, zeolit Y (CBV712) và $N, N, N$-dimethylethyl cyclohexyl ammonium (DMECHA) được thêm vào dung dịch và khuấy thêm 30 phút nữa. Dung dịch thu được có tỉ lệ mol: $26 \mathrm{H}_{2} \mathrm{O}: 0,25 \mathrm{Na}_{2} \mathrm{O}: 1 \mathrm{SiO}_{2}$ : $0,024 \mathrm{Al}_{2} \mathrm{O}_{3}: 0,14$ DMECHA-OH được chuyển vào bình teflon đặt trong autoclave (Parr), được già hóa ở $150^{\circ} \mathrm{C}$ và khuấy liên tục trong 6 ngày. Sản phẩm rắn được tách bằng cách lọc hút chân không, rửa nhiều lần bằng nước đề ion hóa và sấy khô ở $80^{\circ} \mathrm{C}$ trong 20 giờ. Sản phẩm sau khi sấy được nung trong không khí ở nhiệt độ $580^{\circ} \mathrm{C}$ trong 8 giờ với tốc độ gia nhiệt $5^{\circ} \mathrm{C} /$ phút để thu được zeolit SSZ-13.

Tổng hợ Cu-SSZ-13: Zeolit SSZ-13 sau khi nung được trao đổi ion với dung dịch $\mathrm{NH}_{4} \mathrm{NO}_{3}$ $0,5 \mathrm{M}$ ở $80^{\circ} \mathrm{C}$ qua đêm. Mẫu $\left(\mathrm{NH}_{4}-\mathrm{SSZ}-13\right)$ sau 
đó được nung ở $550^{\circ} \mathrm{C}$ trong 4 giờ để chuyển hóa thành H-SSZ-13. Cuối cùng, $3 \%$ trọng lượng đồng (được tính toán với hàm lượng chính xác) được đưa lên zeolit sử dụng muối đồng axetat $\mathrm{Cu}\left(\mathrm{CH}_{3} \mathrm{COO}\right)_{2} \cdot \mathrm{H}_{2} \mathrm{O}$, và sau đó nung ở $550^{\circ} \mathrm{C}$ trong 2 giờ để thu được $\mathrm{Cu}-\mathrm{SSZ}-13$.

Xư lý thủy nhiệt: Cu-SSZ-13 được xử lý thủy nhiệt ở $750^{\circ} \mathrm{C}$ trong 80 giờ trong không khí chứa $10 \% \mathrm{H}_{2} \mathrm{O}$ trong thiết bị phản ứng dòng.

\subsection{Nghiên cứu các đặc trung xúc tác}

Các mẫu nhiễu xạ tia X (XRD) được thu thập ở nhiệt độ phòng trên máy đo nhiễu xạ Bruker $\mathrm{D} 8$ sử dụng bức xạ $\mathrm{Cu} \mathrm{K} \alpha(\lambda=1,5418 \AA \AA$ ). Dũ liệu được thu thập với góc quét $2 \theta$ từ $5,0^{\circ}$ đến $40^{\circ}$ (hoặc $50,0^{\circ}$ ) với bước quét $0,02^{\circ}$ và thời gian 2 giây mỗi bước. Hình thái hạt được xác định bằng phương pháp hiển vi điện tử quét (SEM), và thành phần nguyên tố của zeolit được phân tích bằng cách sử phương pháp phổ tán sắc năng lượng tia $\mathrm{X}(\mathrm{EDX})$ trên cùng thiết bị JEOL JSM7400F, hoạt động ở điện áp gia tốc 3-10 $\mathrm{keV}$, và dòng điện $10 \mu \mathrm{A}$. Thể tích vi mao quản và diện tích bề mặt của các chất xúc tác zeolit được đo bằng $\mathrm{N}_{2}$ ở $-196^{\circ} \mathrm{C}$ trên thiết bị Micromerencies 3Flex. Trước khi đo các chất hấp phụ, mỗi mẫu được loại bỏ hơi nước và khử khí ở nhiệt độ $350^{\circ} \mathrm{C}$ trong 6 giờ.

\subsection{Phản ứng khử chọn lọc $\mathrm{NO}_{x}$ bằng $\mathrm{NH}_{3}$}

Các mẫu bột của chất xúc tác zeolit chưa và đã già hóa được ép, nghiền và rây với kích cỡ hạt từ 0,25 đến $0,35 \mathrm{~mm}$. Sau đó, $0,3 \mathrm{~g}$ của mỗi chất xúc tác được nạp vào một lò phản ứng ống kim loai đường kính $1 \mathrm{~cm}$. Thành phần khí nạp được điều khiển bởi các bộ điều khiển dòng chảy của mỗi dòng khí với thành phần là 500 ppm $\mathrm{NH}_{3}$, 500 ppm NO, $5 \% \mathrm{H}_{2} \mathrm{O}, 15 \% \mathrm{O}_{2}, 5 \% \mathrm{CO}_{2}$ trong $\mathrm{N}_{2}$ trong khoảng nhiệt độ từ 150 đến $500^{\circ} \mathrm{C}$. Thực nghiệm đánh giá hoạt tính SCR được thực hiện ở áp suất khí quyển và vận tốc dòng theo giờ (GHSV) không đổi theo giờ là $90000 \mathrm{~h}^{-1}$. Thành phần khí ra được đo bằng bộ phân tích khí FTIR MKS Multigas 2030HS.

\section{Kết quả và thảo luận}

\section{1. Đặc trung xúc tác}

Phổ nhiễu xạ XRD của zeolit SSZ-13 và $\mathrm{Cu}$ SSZ-13 (Hình 3) cho thấy vật liệu tổng hợp có độ kết tinh cao và độ tinh thể tốt, không phát hiện sự có mặt của tạp chất hoặc pha tinh thể thứ cấp. Tất cả các đỉnh nhiễu xạ đều phù hợp với các mẫu vật liệu zeolit $\mathrm{CHA}$ đã được công bố trước đây $[19,24]$.

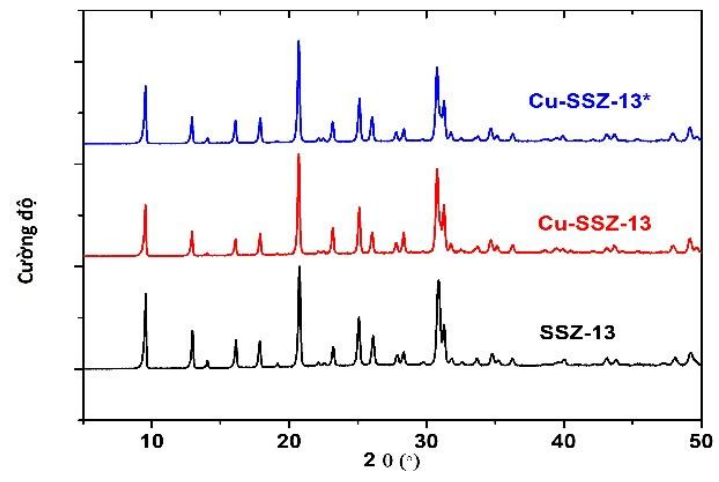

Hình 3. Phổ nhiễu xạ tia X của SSZ-13 $(\mathrm{Si} / \mathrm{Al}=10), \mathrm{Cu}-\mathrm{SSZ}-13$, và $\mathrm{Cu}-\mathrm{SSZ}-13 *$.

Ảnh SEM (Hình 4) cho thấy hình thái của hạt zeolit SSZ-13 (hoặc Cu-SSZ-13) có dạng hình thoi, với kích thước hạt đồng đều xấp xỉ 500-700 $\mathrm{nm}$. Tỉ lệ $\mathrm{Si} / \mathrm{Al}=10$ được xác định bằng phương pháp EDX. Zeolit SSZ-13 thuộc loại zeolit có hàm lượng $\mathrm{Si}$ cao nên rất bền nhiệt vì liên kết Si$\mathrm{O}$ bền vững hơn liên kết Al-O.

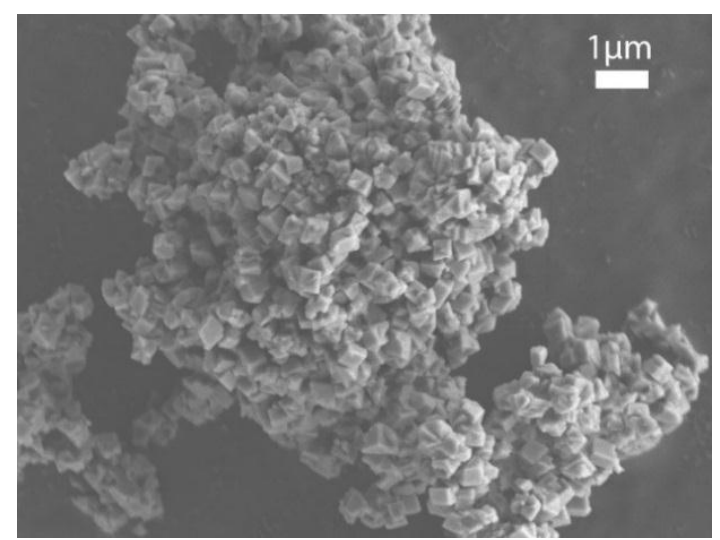

Hình 4. Hình ảnh SEM của mẫu SSZ-13. 
Phương pháp hấp phụ $\mathrm{N}_{2}$ có vai trò quan trọng trong việc so sánh chất xúc tác được nghiên cứu so với các vật liệu xúc tác khác. Đường hấp phụ đẳng nhiệt của SSZ-13 và $\mathrm{Cu}$-SSZ-13 ở $196^{\circ} \mathrm{C}$ (Hình 5) thuộc phân loại I của IUPAC, đặc trưng cho vật liệu vi mao quản.

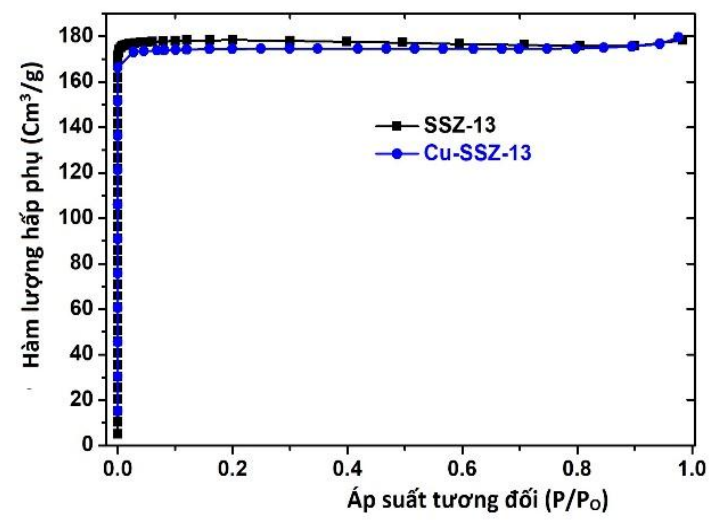

Hình 5. Đường đẳng nhiệt hấp phụ $\mathrm{N}_{2}$ của SSZ-13 và $\mathrm{Cu}-\mathrm{SSZ}-13$ ở $^{-196^{\circ} \mathrm{C} \text {. }}$

Cả 2 mẫu đều hấp phụ mạnh $\mathrm{N}_{2}$ ở áp suất thấp và đạt đến độ hấp phụ bão hòa $\left(\sim 180 \mathrm{~cm}^{3} / \mathrm{g}\right)$ tương đối nhanh $(\mathrm{P} / \mathrm{Po}<0,1)$, cho thấy hai vật liệu có thể tích vi mao quản lớn, là đặc trưng quan trọng của xúc tác dị thể. Kết quả về diện tích bề mặt được tính toán theo phương pháp BrunauerEmmett-Teller (BET) và thể tích mao quản được tính theo phương pháp $\mathrm{t}$-plots được tóm tắt trong Bảng 1.

Bảng 1. Diện tích bề mặt và thể tích mao quản của zeolit SSZ-13 và $\mathrm{Cu}-\mathrm{SSZ}-13$

\begin{tabular}{lll}
\hline Vật liệu & SSZ-13 & Cu-SSZ-13 \\
\hline S_ngoài $\left(\mathrm{m}^{2} / \mathrm{g}\right)$ & 8 & 9 \\
S_MQ $\left(\mathrm{m}^{2} / \mathrm{g}\right)$ & 570 & 542 \\
V_MQ $\left(\mathrm{cm}^{3} / \mathrm{g}\right)$ & 0,27 & 0,27 \\
\hline
\end{tabular}

*S_ngoài: diện tích ngoài mao quản (external surface area), S_MQ: diện tích trong mao quản, V_MQ: thể tích mao quản

\subsection{Hoạt tính xúc tác}

Hình 6 cho thấy sự phụ thuộc hoạt tính xúc tác của $\mathrm{Cu}-\mathrm{SSZ}-13$ trong $\mathrm{NH}_{3}-\mathrm{SCR}$ vào nhiệt độ phản ứng trong khoảng 150 đến $500^{\circ} \mathrm{C}$. Độ chuyển hóa $\mathrm{NO}_{x}$ tăng theo nhiệt độ từ 150 $250^{\circ} \mathrm{C}$, đạt mức chuyển hóa gần $100 \%$ ở nhiệt độ từ $250-320^{\circ} \mathrm{C}$, đó là đặc điểm điển hình trong các chất xúc tác $\mathrm{Cu}$-zeolit SCR [10,25-28]. Độ chuyển hóa $\mathrm{NO}_{\mathrm{x}}$ bắt đầu giảm ở nhiệt độ trên $350^{\circ} \mathrm{C}$, được cho là do hoạt tính ngày càng tăng của phản ứng oxy hóa $\mathrm{NH}_{3}$ cạnh tranh với phản ứng SCR ở nhiệt độ cao [6].

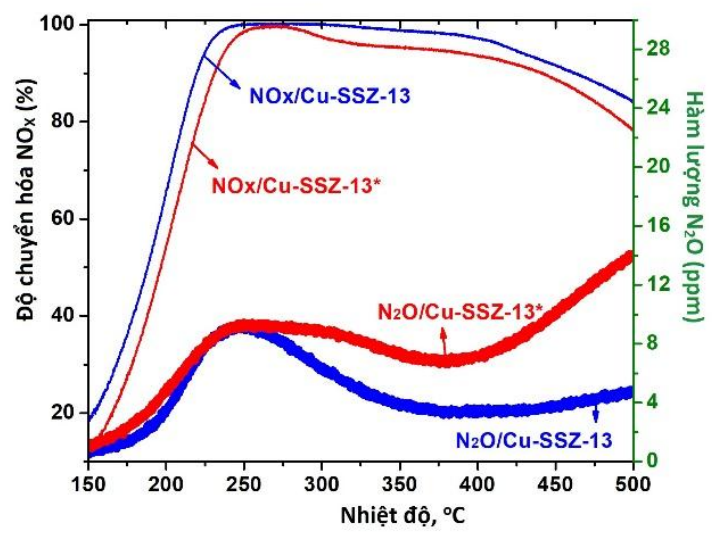

Hình 6. Độ chuyển hóa $\mathrm{NO}_{\mathrm{x}}\left(\mathrm{NO}_{\mathrm{x}}\right.$ conversion, \%, trục trái) và hàm lượng sản phẩm phụ $\mathrm{N}_{2} \mathrm{O}(\mathrm{ppm}$, trục phải) theo nhiệt độ phản ứng (Inlet gas temperature) trên xúc tác Cu-SSZ-13 ban đầu (fresh) và sau khi xử lý thủy nhiệt xúc tác $\mathrm{Cu}-\mathrm{SSZ}-13$ * (hydrothermal treatment) Điều kiện phản ứng: 500 ppm $\mathrm{NH}_{3}, 500 \mathrm{ppm} \mathrm{NO}, 5 \% \mathrm{H}_{2} \mathrm{O}, 15 \% \mathrm{O}_{2}$, $5 \% \mathrm{CO}_{2}$ and balance $\left.\mathrm{N}_{2}, \mathrm{GHSV}=90,000 \mathrm{~h}^{-1}\right)$.

$\mathrm{N}_{2} \mathrm{O}$ là sản phẩm phụ không mong muốn của phản ứng $\mathrm{NH}_{3}-\mathrm{SCR}$, vì khả năng gây hiệu ứng nhà kính cao $\left(\sim 200\right.$ lần so với $\left.\mathrm{CO}_{2}\right)$ và nó tạo ra một chất làm ảnh hưởng xấu đến tầng ozone [29]. Nồng độ $\mathrm{N}_{2} \mathrm{O}$ được tạo ra trên xúc tác $\mathrm{Cu}-\mathrm{SSZ}$ 13 cũng theo một xu hướng tương tự sự chuyển hóa $\mathrm{NO}_{\mathrm{x}}$, đạt nồng độ cao nhất ở khoảng $250^{\circ} \mathrm{C}$. Trên $350^{\circ} \mathrm{C}$, nồng độ $\mathrm{N}_{2} \mathrm{O}$ bắt đầu tăng trở lại theo nhiệt độ, có thể do tốc độ phản ứng oxi hóa $\mathrm{NH}_{3}$ tăng ở nhiệt độ cao [26]. Sự hình thành $\mathrm{N}_{2} \mathrm{O}$ trên xúc tác $\mathrm{Cu}-\mathrm{SSZ}-13$ trước khi xử lý thủy nhiệt cho thấy hàm lượng $\mathrm{N}_{2} \mathrm{O}$ trên zeolit $8 \mathrm{MR}$ này $(\sim 10$ ppm) thấp hơn nhiều so với zeolit MQ trung bình và lớn ( 20 ppm) [30-32].

Để khảo sát độ bền thủy nhiệt, $\mathrm{Cu}-\mathrm{SSZ}-13$ được xử lý trong không khí với $10 \% \mathrm{H}_{2} \mathrm{O}$ ở 
$750^{\circ} \mathrm{C}$ trong 80 giờ (kí hiệu sau khi xử lý thủy nhiệt là $\left.\mathrm{Cu}-\mathrm{SSZ}-13^{*}\right)$. Kết quả nhiễu xạ tia X (Hình 3) cho thấy độ tinh thể của xúc tác được giữ nguyên sau khi xử lý thủy nhiệt ở $750^{\circ} \mathrm{C}$. Hoạt tính của $\mathrm{NH}_{3}-\mathrm{SCR}$ của $\mathrm{Cu}-\mathrm{SSZ}-13^{*}$ được thể hiện trên Hình 6. Hiệu suất chuyển hóa giảm nhẹ đến vừa phải trên toàn bộ cửa sổ nhiệt độ phản ứng được quan sát thấy sau khi già hóa $750^{\circ} \mathrm{C}$ trong 80 giờ. Nhìn chung, sự hình thành $\mathrm{N}_{2} \mathrm{O}$ ở nhiệt độ thấp tương đương sau khi xử lý thủy nhiệt ở $750^{\circ} \mathrm{C}$, trong khi hàm lượng $\mathrm{N}_{2} \mathrm{O}$ tăng ở nhiệt độ cao so với mẫu Cu-SSZ-13 ban đầu. Kết quả khảo sát cũng cho thấy vật liệu tổng hợp có độ chuyển hóa gần tương đương với xúc tác thương mại hóa chabazite $(\mathrm{Cu}-\mathrm{CHA}$ hoặc Cu-SSZ-13 thương mại) $[4,8,30]$.

\section{Kết luận}

Zeolit SSZ-13 ( $\mathrm{Si} / \mathrm{Al}=10)$ được tổng hợp thành công với cấu trúc $\mathrm{CHA}$ (CHA framework) khi sử dụng cation định hướng cấu trúc $N, N, N$ dimethylethylcyclohexylammonium với chi phí thấp hơn TMAda ${ }^{+}$. Vật liệu tổng hợp được có độ tinh thể cao, diện tích và thể tích mao quản lớn. Quan trọng hơn, zeolit $\mathrm{Cu}-\mathrm{SSZ}-13$ thể hiện các tính chất hóa học gần tương đương như vật liệu thương mại $\mathrm{Cu}-\mathrm{CHA} / \mathrm{Cu}-\mathrm{SSZ}-13$, với độ chuyển hóa $\mathrm{NO}_{\mathrm{x}}$ đạt gần $100 \%$ ở $250^{\circ} \mathrm{C}$, lượng $\mathrm{N}_{2} \mathrm{O}$ hình thành thấp $(\sim 10 \mathrm{ppm})$, và độ bền thủy nhiệt tương đối cao. Sự kết hợp của hoạt tính xúc tác cao, chi phí tổng hợp thấp mang đến cơ hội cho các ứng dụng rộng rãi của zeolit SSZ-13 này trong các lĩnh vực hấp phụ, tách và xúc tác trong tương lai, đặc biệt cho xử lý khí thải $\mathrm{NO}_{\mathrm{x}}$ từ động cơ diesel.

\section{Lời cảm ơn}

Nghiên cứu này được tài trợ bởi Đại học Quốc gia Hà Nội trong đề tài mã số QG.19.09.

Nhóm tác giả xin cảm ơn Đại học Delaware, Hoa Kỳ đã giúp đỡ thực hiện một số thí nghiệm phản ứng.

\section{Tài liệu tham khảo}

[1] Tian Peng, Wei Yingxu, Ye Mao, Liu Zhongmin, Methanol to Olefins (MTO): From Fundamentals to Commercialization. ACS Catalysis 5(3) (2015) 1922-1938. https://doi.org/10.1021/acscatal.5b00007.

[2] D. Chen, K. Moljord, A. Holmen, A methanol to olefins review: Diffusion, coke formation and deactivation on SAPO type catalysts. Microporous and Mesoporous Materials 164 (2012) 239-250. https://doi.org/10.1016/j.micromeso.2012.06.046.

[3] Yashodhan Bhawe, Manuel Moliner-Marin, Jonathan D. Lunn, Yu Liu, Andrzej Malek, Mark Davis, Effect of Cage Size on the Selective Conversion of Methanol to Light Olefins. ACS Catalysis 2(12) (2012) 2490-2495. https:// doi.org/10.1021/cs300558x.

[4] Feng Gao, Ja Hun Kwak, Janos Szanyi, Charles H. F. Peden, Current Understanding of CuExchanged Chabazite Molecular Sieves for Use as Commercial Diesel Engine DeNOx Catalysts. Topics in Catalysis 56(15-17) (2013) 1441-1446. https://doi.org/10.1007/s11244-013-0145-8.

[5] S. Brandenberger, O. Kröcher, A. Tissler, R. Althoff, The State of the Art in Selective Catalytic Reduction of NOx by Ammonia Using MetalExchanged Zeolite Catalysts. Catalysis Reviews 50(4) (2008) 493-498. https://doi.org/10.1080/01 614940802480122.

[6] A.M. Beale, F. Gao, I. Lezcano-Gonzalez, C.H.F. Peden, J. Szanyi, Recent advances in automotive catalysis for NOx emission control by small-pore microporous materials. Chemical Society Reviews 44(20) (2015) 7371-7378. https://doi.org/10.1039/ C5CS00108K

[7] C. Paolucci, J.R. Di lorio, F.H. Ribeiro, R. Gounder, W.F. Schneider, Catalysis Science of NOx Selective Catalytic Reduction With Ammonia Over Cu-SSZ-13 and Cu-SAPO-34. Advances in Catalysis 59 (2016) 5-16. https://doi. org/10.1016/bs.acat.2016.10.002.

[8] Guan Bin, Zhan Reggie, Lin He, Huang Zhen, Review of state of the art technologies of selective catalytic reduction of NOx from diesel engine exhaust. Applied Thermal Engineering 66(1) (2014) 396-411. https://doi.org/10.1016/j. applther maleng.2014.02.021.

[9] J. Steven Schmieg, H. Se Oh, H. Chang Kim, B. David Brown, H. Jong Lee, H.F. Charles Peden, Do Heui Kim, Thermal durability of $\mathrm{Cu}-\mathrm{CHA}$ $\mathrm{NH}_{3}$-SCR catalysts for diesel NOx reduction. Catalysis Today 184(1) (2012) 252-253. https:// doi.org/10.1016/j.cattod.2011.10.034. 
[10] Taekyung Ryu, Nak Ho Ahn, Seungwan Seo, Jung Cho, Hyojun Kim, Donghui Jo, Gi Tae Park, Pyung Soon Kim, Chang Hwan Kim, Elliott L. Bruce, Paul A. Wright, In-Sik Nam, and Suk Bong Hong, Fully Copper-Exchanged HighSilica LTA Zeolites as Unrivaled Hydrothermally Stable $\mathrm{NH}_{3}$-SCR Catalysts. Angewandte Chemie International Edition 56(12) (2017) 3256-3258. https://doi.org/10.1002/anie.201610547.

[11] Manuel Moliner, Cristina Franch, Eduardo Palomares, Marie Grill and Avelino Corma, $\mathrm{Cu}-$ SSZ-39, an active and hydrothermally stable catalyst for the selective catalytic reduction of NOx. Chemical Communications 48(66) (2012) 8264-8266. https://doi.org/10.1039/C2CC33992G.

[12] Nuria Martín, Cristian R. Boruntea, Manuel Moliner, Avelino Corma, Efficient synthesis of the Cu-SSZ-39 catalyst for DeNO $_{x}$ applications. Chemical Communications 51(55) (2015) 1103111032. https://doi.org/10.1039/C5CC03200H.

[13] Kim Young Jin, Lee Jun Kyu, Min Kyung Myung, Hong Suk Bong, Nam In-Sik, Cho Byong K., Hydrothermal stability of CuSSZ13 for reducing $\mathrm{NO}_{\mathrm{x}}$ by $\mathrm{NH}_{3}$. Journal of Catalysis 311 (2014) 447. https://doi.org/10.1016/j.jcat.2013.12.012.

[14] D.W. Fickel, R.F. Lobo, Copper Coordination in $\mathrm{Cu}-\mathrm{SSZ}-13$ and $\mathrm{Cu}-\mathrm{SSZ}-16$ Investigated by Variable-Temperature XRD. The Journal of Physical Chemistry C 114(3) (2010) 1633-1640. https://doi.org/10.1021/jp9105025.

[15] Takushi Sonoda, Toshihiro Maruo, Yoshitaka Yamasaki, Nao Tsunoji, Yasuyuki Takamitsu, Masahiro Sadakane and Tsuneji Sano, Synthesis of high-silica AEI zeolites with enhanced thermal stability by hydrothermal conversion of FAU zeolites, and their activity in the selective catalytic reduction of NOx with NH3. Journal of Materials Chemistry A 3(2) (2015) 857-865. https://doi.org/ 10.1039/C4TA05621C.

[16] G. Blakeman Philip, M. Burkholder Eric, Chen Hai-Ying, E. Collier Jillian, M. Fedeyko Joseph, Jobson Hoi, R. Rajaram Raj, The role of pore size on the thermal stability of zeolite supported $\mathrm{Cu}$ SCR catalysts. Catalysis Today 231(2014) 56-63. https://doi.org/10.1016/j.cattod.2013.10.047.

[17] C.M. Baerlocher, L.B. Database of Zeolite Structures. http://www.iza-structure.org/databases/, 2017.

[18] J.V. Smith, Crystal structures with a chabazite framework. I. Dehydrated Ca-chabazite. Acta Crystallographica 15(9) (1962) 838-843. https:// doi.org/10.1016/j.micromeso.2013.07.033.
[19] S.I. Zones, Conversion of faujasites to high-silica chabazite SSZ-13 in the presence of $\mathrm{N}, \mathrm{N}, \mathrm{N}-$ trimethyl-1-adamantammonium iodide. Journal of the Chemical Society, Faraday Transactions 87(22) (1991) 3710-3715. https://doi.org/ 10.1039/ FT9918703709.

[20] Ruinian Xu, Runduo Zhang, Ning Liu, Biaohua Chen, and Shi Zhang Qiao, Template Design and Economical Strategy for the Synthesis of SSZ-13 (CHA-Type) Zeolite as an Excellent Catalyst for the Selective Catalytic Reduction of NOx by Ammonia. ChemCatChem 7(23) (2015) 38423843. https://doi.org/10.1002/cctc.201500771.

[21] G. Cao, J.F. Brody, M.J. Shah, Light olefin selective oxygenate conversion process using CHA framework type aluminosilicate. https://patents. google.com/patent/US7772335B1/en, 2010.

[22] Guang Cao, Machteld M. Mertens, Anil S. Guram, Hailian Li, Jeffrey C. Yoder, Synthesis of chabazite-containing molecular sieves and their use in the conversion of oxygenates to olefins. https://patents.google.com/patent/US7754187B2/ en, 2012.

[23] D. Trong Pham, R. Matthew Hudson, M. Craig Brown, F. Raul Lobo, Molecular Basis for the High $\mathrm{CO}_{2}$ Adsorption Capacity of Chabazite Zeolites. ChemSusChem 7(11) (2014) 3031-3037. https://doi.org/10.1002/cssc.201402555.

[24] M.A. Camblor, L.A. Villaescusa, M.J. DíazCabañas, Synthesis of all-silica and high-silica molecular sieves in fluoride media. Topics in Catalysis 9(1-2) (1999) 62-65. https://doi.org/10. 1023/A:1019154304344.

[25] G. Delahay, B. Coq, S. Kieger, B. Neveu, The origin of $\mathrm{N}_{2} \mathrm{O}$ formation in the selective catalytic reduction of $\mathrm{NO}_{\mathrm{x}}$ by $\mathrm{NH}_{3}$ in $\mathrm{O}_{2}$ rich atmosphere on $\mathrm{Cu}$-faujasite catalysts. Catalysis Today 54(4) (1999) 434-437. https://doi.org/10.1016/S09205861(99)00206-0.

[26] Norman Wilken, Kurnia Wijayanti, Krishna Kamasamudram, W. Neal Currier, Ramya Vedaiyan, Aleksey Yezerets, Louise Olsson, Mechanistic investigation of hydrothermal aging of $\mathrm{Cu}$-Beta for ammonia SCR. Applied Catalysis B: Environmental 111(2012) 60-61. https://doi. org/10.1016/j.apcatb.2011.09.018.

[27] Jixing Liu, Weiyu Song, Chi Xu, Jian Liu, Zhen Zhao, Yuechang Wei, Aijun Duan and Guiyuan Jiang, The selective catalytic reduction of NOx over a Cu/ZSM-5/SAPO-34 composite catalyst. RSC Advances 5(127) (2015) 104923-104924. https://doi.org/10.1039/C5RA22234F. 
[28] Oana Mihai, R. Catur Widyastuti, Stanislava Andonova, Krishna Kamasamudram, Junhui Li, Saurabh Y. Joshi, Neal W. Currier, Aleksey Yezerets, Louise Olsson, The effect of Cu-loading on different reactions involved in $\mathrm{NH}_{3}-\mathrm{SCR}$ over $\mathrm{Cu}-\mathrm{BEA}$ catalysts. Journal of Catalysis 311 (2014) 174-175. https://doi.org/10.1016/j.jcat.2013.11.016.

[29] A.R. Ravishankara, J.S. Daniel, R.W. Portmann, Nitrous Oxide $\left(\mathrm{N}_{2} \mathrm{O}\right)$ : The Dominant OzoneDepleting Substance Emitted in the 21st Century. Science 326(5949) (2009) 123-125. https://doi. org/10.1126/science.1176985.

[30] Hai-Ying Chen, $\mathrm{Cu} /$ Zeolite SCR Catalysts for Automotive Diesel NOx Emission Control, UreaSCR Technology for deNO $\mathrm{x}_{\mathrm{x}}$ After Treatment of
Diesel Exhausts (2014) 125-127. https://doi.org/ 10.1007/978-1-4899-8071-7_5.

[31] Ja Hun Kwak, G. Russell Tonkyn, Do Heui Kim, János Szanyi, H.F. Charles Peden, Excellent activity and selectivity of $\mathrm{Cu}-\mathrm{SSZ}-13$ in the selective catalytic reduction of $\mathrm{NO}_{x}$ with $\mathrm{NH}_{3}$. Journal of Catalysis 275(2) (2010) 187-189. https://doi.org/10.1016/j.jcat.2010.07.031.

[32] Ja Hun Kwak, G. Russell Tonkyn, Do Heui Kim, János Szanyi, H.F. Charles Peden, A comparative study of $\mathrm{N}_{2} \mathrm{O}$ formation during the selective catalytic reduction of $\mathrm{NO}_{x}$ with $\mathrm{NH}_{3}$ on zeolite supported $\mathrm{Cu}$ catalysts. Journal of Catalysis 329 (2015) 495-497. https://doi.org/10.1016/j.jcat. 2015. 06.016 . 